\title{
Anabases
}

ANABASES Traditions et réceptions de l'Antiquité

$9 \mid 2009$

Varia

\section{Philologie classique et modernité}

\section{Giovanni Leghissa}

\section{OpenEdition}

\section{Journals}

Edizione digitale

URL: http://journals.openedition.org/anabases/518

DOI: 10.4000/anabases.518

ISSN: 2256-9421

\section{Editore}

E.R.A.S.M.E.

\section{Edizione cartacea}

Data di pubblicazione: 1 marzo 2009

Paginazione: 263-266

ISSN: 1774-4296

\section{Notizia bibliografica digitale}

Giovanni Leghissa, « Philologie classique et modernité », Anabases [Online], 9 | 2009, Messo online il 01 mars 2012, consultato il 21 octobre 2019. URL : http://journals.openedition.org/anabases/518 ; DOI : $10.4000 /$ anabases.518 
Anabases 9 (2009), p. 263-266.

\section{Philologie classique et modernité}

GiovanNi LeGHISSA

L'AUTEUR PRÉSENTE SON DERNIER OUVRAGE: Incorporare l'antico. Filologia classica e invenzione della modernità, Milano, Mimesis, 2007, 262 p.

Pur non avendo operato una riflessione sui propri statuti e sulla propria collocazione all'interno dell'enciclopedia dei saperi paragonabile a quella compiuta negli ultimi decenni dall'antropologia culturale, anche la scienza dell'antichità ha mostrato la capacità di interrogarsi sul senso complessivo del proprio discorso-sono ben note le ricostruzioni storiche di alcuni momenti particolarmente rilevanti della storia della disciplina offerte da autori come Momigliano o Canfora. Lo scopo che ho inteso perseguire in Incorporare l'antico non intendeva però essere quello di aggiungere qualcosa ai dati già in nostro possesso sulla storia degli studi classici. In maniera forse più ambiziosa, ho voluto piuttosto porre la domanda seguente: in che senso il sapere sul mondo antico ha costruito un' immagine dell'alterità greca che potesse costituire la base per articolare un discorso nuovo sull'identità europea moderna? Per quali ragioni, in altre parole, i Greci studiati dai filologi sono diventati, in una fase specifica della storia europea, lo specchio che poteva restituire al soggetto europeo un'immagine di sé guardando la quale diveniva possibile definire i confini che separano il medesimo e l'altro?

Il riferimento alla questione dell'alterità non è casuale: a rendere la ricerca condotta in Incorporare l'antico qualcosa di diverso rispetto a una ricostruzione storica della disciplina filologica è precisamente la volontà di porre il materiale storico che viene sottoposto ad analisi in relazione con le questioni teoriche che sono state sollevate, nella seconda metà del Novecento, dalle filosofie della differenza (in primis da Foucault). Inserita in tale contesto, la formazione culturale greca nelle opere di Winckelmann, di Wolf, o di Humboldt non è un semplice oggetto di studio, ma costituisce un oggetto del desiderio, nei confronti del quale è possibile operare un vero e proprio investimento affettivo. Oggetto perduto, ben inteso: la ricostruzione storica del mondo antico, sin dalla storia dell'arte winckelmanniana, non intende annullare la distanza storica tra i moderni e i greci. Ed è precisamente questo il punto che mi interessava maggiormente mettere in luce: la grecità intesa quale luogo dell'origine, ricostruendo la quale è possibile 
conoscere la verità del soggetto moderno, non si aggiunge alla metodica filologica dall'esterno, quasi fosse un supplemento ideologico volto a giustificare il ruolo pedagogico che il filologo si sentiva chiamato a svolgere; piuttosto, è nella stessa messa a punto del metodo filologico che si forma quella discorsività in virtù della quale si postula la coincidenza tra grecità e origine della tradizione occidentale.

Questo è dunque il quadro d'insieme, a partire dal quale ho voluto interrogare alcuni momenti salienti della storia degli studi classici. Va aggiunta, a questo punto, una precisazione essenziale sul valore esemplare da me conferito a ciascuno degli autori che ho scelto di sottoporre ad analisi. La scelta da me compiuta non ha avuto solo la funzione di delimitare un campo di indagine che altrimenti sarebbe risultato troppo vasto. Se ho scelto di dare spazio solo a Winckelmann, a Fréret, alla coppia Wolf-Humboldt e a Nietzsche (lasciando fuori autori come Boeckh o Wilamowitz, tanto per fare due nomi illustri tra quelli che non ho preso in considerazione), ciò è avvenuto per ragioni ben precise. Sono persuaso che ciascun momento della storia degli studi sul mondo antico debba essere analizzato all'interno di un campo di forze intellettuali specifiche, in cui la questione del significato da attribuire al rapporto con la classicità greca viene declinato in rapporto all'esigenza, a sua volta locale e contingente, di definire in un certo modo il confine tra passato e presente, tra il medesimo e l'altro. La filologia tedesca nella linea che va da Wolf a Nietzsche-e che comporta una rottura rispetto alle tendenze presenti nello studio del mondo antico attive nel secolo XVIII-costituisce dunque un esempio tra i molti possibili di un discorso sull'antico la cui portata e la cui struttura andrebbe poi verificata in altri periodi e in altri contesti (per esempio al di fuori dalla Germania), al fine di misurare sul campo le trasformazioni che eventualmente hanno avuto luogo rispetto al paradigma iniziale. Ma in virtù delle caratteristiche che tale esempio paradigmatico presenta, diviene possibile formulare un'ipotesi dalla portata più generale sulla filologia quale ambito disciplinare che ha avuto la funzione di costruire un elemento importante dell'identità europea, dal momento che gli autori da me presi in considerazione permettono una chiarificazione di alcune delle strutture portanti del discorso moderno sull'antichità.

Di particolare rilievo è il modo in cui la disciplina filologica giustifica se stessa non solo e non tanto quale discorso scientifico sul passato, ma anche quale impresa politico-pedagogica. Attraverso la fondazione metodologica dell'Altertumswissenschaft, Wolf non si limita infatti ad articolare uno specifico ambito disciplinare, la cui estensione è rimasta, grosso modo, la stessa sino ad oggi, ma definisce anche i compiti che ha il filologo in quanto educatore. In questo senso, si può dire che Wolf non sia stato tanto il fondatore di una disciplina (dal secolo XVII ormai esistevano un po'ovunque cattedre di studi filologici), quanto l'istitutore di una forma di discorsività. Tale discorsività si fonda su due assunti, apparentemente in contraddizione tra loro. Da un lato, si ha il riconoscimento del fatto che la distanza storica che separa il presente europeo moderno dal passato greco sia tale rendere il mondo greco irrimediabilmente perduto. D'altro lato, alla filologia viene attribuito il compito di restituire allo spirito greco una nuova patria in cui poter continuare a svolgere le funzioni che svolgeva nel mondo antico, ovvero formare cittadini autonomi e responsabili. La contraddizione, dicevo, è solo apparente, perché per Wolf la saldatura tra il momento storico-ricostruttivo e il momento pedagogico-formativo avviene a partire dall'idea che lo spirito capace di animare le forme espressive della cultura greca, dalla letteratura all'arte, dalla filosofia alla religione, sia identico al Geist tedesco. Il punto è decisivo: non credo infatti si possa comprendere nulla della filologia tedesca inaugurata da Wolf se non si coglie in che senso la triangolazione Kultur/Bildung/Geist giochi un ruolo essenziale all'interno del processo di 
autocostituzione della disciplina filologica. I greci studiati dal filologo hanno un valore esemplare rispetto ad altri popoli del mondo antico (persiani, egizi, ebrei, ecc) perché quella greca è una geistige Kultur, una cultura spirituale. Ora, se a comprendere i Greci sono chiamati i Tedeschi, tra tutte le nazioni europee, ciò accade perché tra il Geist tedesco è quello greco vi è una profonda affinità. Di conseguenza, il lavoro del filologo avrà la funzione di far passare dalla potenza all'atto, attraverso l'incontro con le opere spirituali dell'antichità, tutto ciò che il Geist tedesco contiene in potenza, e ciò in relazione a sfere decisive per il perfezionamento dell'individualità quali l'amore per il sapere e per la libertà, la creatività, l'immaginazione.

E importante altresì notare che, in virtù della propria impostazione, Wolf prende distanza da due approcci al mondo greco che nel secolo xvIII ebbero un notevole peso nella vita intellettuale non solo tedesca, ma anche europea. Da un lato, con Wolf diviene impossibile inserire la cultura greca entro il più vasto contesto del Mediterraneo antico, come invece avveniva per quegli studiosi francesi che, riuniti all'interno dell'Académie des Inscriptions et Belles Lettres, avevano dato, nel contesto europeo di allora, i maggiori contributi alla conoscenza del mondo classico. Per un autore come Fréret (che nel mio libro scelgo come rappresentativo di un'intera tradizione di studi) isolare la cultura greca dalla altre con le quali essa era in contatto, e attribuire ai soli Greci il valore dell'esemplarità, sarebbe stato impossibile. In Wolf, invece, precisamente l'esclusione degli altri popoli dell'antichità dallo sguardo dello storico costituisce il punto di partenza della giustificazione che la filologia classica fornisce di se stessa quale campo disciplinare autonomo. Tuttavia, ciò non deve farci dimenticare che, dal punto di vista metodologico (valore di verità dei risultati raggiunti attraverso la ricerca, analisi della differenza tra certezza e probabilità in campo filologico, esame delle fonti e della loro attendibilità, eccetera), Wolf non fa che ripetere le acquisizioni a cui erano giunti i suoi predecessori francesi un cinquantennio prima. Il fatto che la filologia, con Wolf, si presenti sulla scena della storia come una disciplina specificamente tedesca va allora spiegato con la volontà, da parte di Wolf, di costruire una discorsività di tipo nuovo, in cui l'impegno scientifico si trovava a essere inestricabilmente mescolato a intenti di natura politico-pedagogica.

D'altro lato, una seconda presa di distanza rispetto a quanto era avvenuto in precedenza nell'ambito degli studi sul mondo antico si attua nei confronti della storia dell'arte winckelmanniana. Agli occhi del filologo abituato ormai a servirsi di un metodo rigoroso di analisi dei testi, un lavoro come quello di Winckelmann doveva apparire irrimediabilmente segnato dal dilettantismo e dall'approssimazione (già Heyne, il maestro di Wolf, aveva mosso delle pesanti critiche al metodo storico winckelmanniano). Ma non è solo questione di metodi e di rigore scientifico: l'immedesimazione con i Greci proposta da Winckelmann doveva essere messa da parte per far posto a una forma di incontro con la grecità che passasse attraverso il confronto testuale e la mediazione della scrittura. Certo, la storia dell'arte trova pure il suo posto nell'enciclopedia filologica proposta da Wolf. Ma non la contemplazione estetica, bensì il rigore della critica doveva permettere di far rivivere lo spirito greco. Da qui la necessità di mostrare come il discorso filologico si ponga in alternativa a tutte quelle forme di riappropriazione del mondo classico che invece ponevano l'esemplarità dei Greci nella sola produzione artistica. All'utopia estetica, che per esempio nelle Briefe über die ästhetische Erziehung di Schiller aveva il proprio manifesto programmatico, Wolf intese sostituire una sorta di utopia filologica, che mirava a formare cittadini liberi e responsabili attraverso lo studio dell'antichità mediato dal sapere filologico. Anche in questo caso, però, sarebbe erroneo fermarsi solamente alla rottura compiuta da Wolf. A mio avviso, è importante mettere in luce anche una sorta di continuità sotterranea tra il discorso 
del filologo e quel discorso sul classico che, in Winckelmann, aveva trovato il proprio punto di partenza nella contemplazione del bello. Nella storia dell'arte wincklemanniana trova infatti espressione un paradosso da cui il filologo tenta di liberarsi, ma che in realtà opera segretamente anche nel discorso filologico. In Winckelmann il desiderio di riappropriarsi della classicità per permettere di portare a compimento, attraverso tale riappropriazione, quanto di più nobile e di più compiuto vi è nell'umano, trova sì espressione attraverso una retorica che il filologo, da Wolf in avanti, tenterà poi di attutire, ma tale desiderio di riappropriazione, accompagnato dall'idea che solamente la grecità incarna la perfezione dell'umano, non abbandonerà mai il discorso del filologo sulla grecità. E questo perché (e ciò anche al di fuori del contesto tedesco) alla filologia viene comunque attribuita la funzione di formare coloro che, in virtù della propria appartenenza all'élite borghese, avevano il compito di rappresentare la parte migliore dell'umanità.

Da qui la scelta di concludere la trattazione con un capitolo dedicato a Nietzsche. Quest'ultimo infatti è stato colui che per primo ha visto-e denunciato in modo molto netto-il nesso che lega lo studio della grecità alla volontà di legittimare la presunta superiorità del borghese europeo che, forte dell'affinità "spirituale» tra sé e i Greci, intende occupare una posizione di assoluto privilegio in seno alla storia universale. Non che Nietzsche abbia inteso rinunciare del tutto alla vocazione pedagogica che il filologo, da Wolf in poi, si era assunto. Anche Nietzsche, a modo suo, intendeva porre la propria opera al servizio di un progetto di rinnovamento della Germania di allora-e anche per lui il passaggio attraverso la Grecia costituiva un momento obbligato del proprio percorso. Solo che ai suoi occhi andava radicalmente modificata l'immagine della Grecia veicolata dal sapere filologico. La Grecia di Nietzsche non era infatti la patria dell'armonia e della bellezza, ma costituiva quella fase della tradizione occidentale in cui gli umani avevano saputo confrontarsi in maniera esemplare con le forze oscure della vita, ovvero con tutto ciò che la borghesia filistea dei suoi tempi intendeva cancellare dalla propria esperienza.

Tuttavia è in un'altra direzione che va cercata l'importanza del discorso di Nietzsche sulla filologia. Nietzsche è stato capace di valutare con precisione l'impossibilità di separare in modo netto il piano epistemico che definisce il raggio d'azione di una data disciplina da una serie di interessi di natura non teoretica che invece contribuiscono a legare quella disciplina al contesto più ampio del mondo della vita. Ed è bene tenere sempre presente che l'intera riflessione di Nietzsche sul rapporto che lega il sapere ai bisogni vitali dell'uomo ha avuto il proprio punto di partenza proprio nella riflessione da lui compiuta sullo statuto della filologia. Quest' ultima ha costituito per Nietzsche una sorta di esempio negativo, in quanto proprio il filologo, avendo costruito un'immagine monumentale della Grecia, ha abdicato al compito-irrinunciabile tanto per Nietzsche quanto per Wolf-di indicare ai propri contemporanei qual è la via che conduce a quel poco di felicità terrena che è concesso agli umani.

Giovanni LeGhisSA

Université de Trieste

Pendice Scoglietto 8

I-34127 Trieste

giovanni.leghissa@yahoo.it 